\title{
Frauen häufiger von Herz-Kreislauf-Tod betroffen
}

In Deutschland sterben etwa 36\% aller Männer an Herz-Kreislauf-Erkrankungen. Bei Frauen beträgt der Anteil 44\%. Nach den Wechseljahren nimmt die Gefahr zu. Frauen erleiden zwar seltener einen Herzinfarkt als Männer. Todesfälle durch Herzschwäche kommen jedoch doppelt so häufig vor. An den Spätfolgen von Bluthochdruck auf das Herz sterben Frauen sogar dreimal häufiger als Männer. „Auf geschlechtsspezifische Risikofaktoren allein lassen sich diese Unterschiede nicht zurückführen“, sagt Prof. Martin Hausberg, Karlsruhe, Vorstandsvorsitzender der Deutschen Hochdruckliga.

Denn die meisten Erkrankungen treten erst nach den Wechseljahren auf. Dann nehmen viele Frauen zu. Dabei zeigen neue Studien, dass gesunde Ernährung und Bewegung für Frauen sogar mehr Schutz bieten als für Männer. Hausberg betont: „Hier wäre eine stärkere Prävention von Herz-Kreislauf-Erkrankungen wichtig und nachgewiesenermaßen wirksam“. Auch Rauchen scheint Frauen mehr zu schaden als Männern. Dies könnte nach Einschätzung des Experten an einer höheren Empfindlichkeit der
Schleimhaut in den Atemwegen, aber auch an unterschiedlichen Rauchgewohnheiten liegen: „Frauen fällt es insbesondere im Alter schwerer, das Rauchen aufzugeben“, erläutert Hausberg.

Unterschiedliche Behandlung von Frauen und Männern

In der Behandlung der Risikofaktoren für Herz-Kreislauf-Erkrankungen gibt es schwer erklärbare Unterschiede zwischen Männern und Frauen: Ärzte verordnen Frauen häufiger Diuretika und Betablocker zur Hochdrucktherapie. Mittel gegen hohe Cholesterinwerte bleiben dagegen eher Männern vorbehalten. Dies entspreche aber nicht den Empfehlungen, die auf Risiko-Scores wie dem PROCAM-Score beruhen, meint Hausberg. Der PROCAM Risiko-Score erlaubt es, das Risiko einzuschätzen, innerhalb der nächsten 10 Jahre einen Herzinfarkt zu erleiden. Dabei beruht die Risikobestimmung auf den Daten der Prospective Cardiovascular Münster (PROCAM-) Studie. Allerdings berücksichtigt dieser Score zwar das Geschlecht der Patienten, nicht aber die soziale Herkunft, aus der

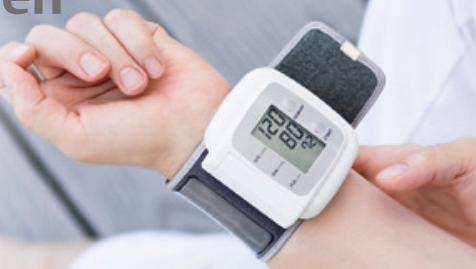

ธ్ für Frauen eine weitere Benachteiligung entstehen kann.

Und auch den Bluthochdruck betreffend - als ein Hauptrisikofaktor für Herzinfarkt und Schlaganfall - unterscheiden sich Männer und Frauen deutlich. Jüngere Frauen haben zwar seltener einen erhöhten Blutdruck als Männer. Im Alter kehrt sich das Verhältnis jedoch um. Sie sind dann auch stärker von Herz-Kreislauf-Erkrankungen betroffen. „Eine rechtzeitige Diagnose der Hochdruckerkrankung ist deshalb bei Frauen besonders wichtig“, sagt Hausberg. Er empfiehlt neben der Messung des Blutdrucks zu Hause auch die ärztlich angeleitete 24-Stunden-Messung, um zusätzliche Anhaltspunkte für die Therapie zu erhalten.

Deutsche Hochdruckliga, 1.12.2014

\section{Erhöhtes Erkrankungsrisiko bei unspezifischem Beschwerdekomplex}

Menschen, die unter einer Vielzahl unspezifischer körperlicher Beschwerden leiden, haben ein erhöhtes Risiko, an Typ-2-Diabetes zu erkranken. Der Beschwerdekomplex ist dabei ein unabhängiger Risikofaktor und nicht mit den klassischen Diabetes-Risikofaktoren assoziiert, berichten Wissenschaftler des Helmholtz Zentrums München im Fachjournal ,BMC Endocrine Disorders ' [BMC Endocrine Disorders, doi:10.1186/1472-6823-14-87].

Unspezifische körperliche Symptome sind häufig erste Anzeichen einer chronischen Erkrankung. Dazu zählen Zeichen einer vitalen Erschöpfung, wie Müdigkeit, Muskelschwäche, Gelenk- und Gliederschmerzen, Schlafstörungen oder Kopfschmerzen. Meist treten sie als Beschwerdekomplex auf, der bereits als ein frühes Warnzeichen für eine Erkrankung der Herzkranzgefäße (koronare Herzerkrankung) gilt. Der Zusammenhang unspezifischer Beschwerden mit Diabetes war bislang unklar.

\section{Symptomkomplex erhöht \\ Diabetesrisiko}

Das Team um Jens Baumert und KarlHeinz Ladwig von der AG Mental Health des Instituts für Epidemiologie II am Helmholtz Zentrum München wertete in Zusammenarbeit mit Johannes Kruse von der Klinik für Psychosomatische Medizin und Psychotherapie des Universitätsklinikums Gießen und Marburg und Kollegen der Klinik und Poliklinik für Psychosomatische Medizin und Psychotherapie der TU München dazu bevölkerungsbasierte Daten von etwa 10000 Teilnehmern der MONICA/KORA-Kohortenstudie aus. Unspezifische körperliche Beschwerden wurden anhand eines Score-Systems erfasst. Pro Score-Wert stieg das Risiko, an Typ-2-Diabetes zu erkranken, um $2 \%$ an.
Diese Risikoerhöhung war unabhängig von klassischen Diabetes-Risikofaktoren, wie Übergewicht, Bluthochdruck oder Rauchen.

Unabhängiger Risikofaktor für die Früherkennung von Diabetes „Unsere Ergebnisse zeigen eine deutliche Risikoerhöhung für Typ-2-Diabetes, wenn ein unspezifischer Symptomkomplex vorliegt“, sagt Erstautor Baumert. „Daher sollten solche Symptommuster als mögliche Warnzeichen in der Früherkennung dieser Stoffwechselerkrankung berücksichtigt werden.“

Ziel des Helmholtz Zentrums München, Partner im Deutschen Zentrum für Diabetesforschung (DZD), ist es, neue Ansätze für Diagnose, Therapie und Prävention der großen Volkskrankheiten, wie Diabetes mellitus, zu entwickeln.

Helmholtz Zentrum München, 1.12.2014 
Klauber, Geraedts, Friedrich, Wasem (Hrsg.). Krankenhaus-Report 2014. Schwerpunktthema „Patientensicherheit““. Stuttgart: Schattauer-Verlag 2014, 528 S., 83 Abb., 64 Tab., kart. Mit Online-Zugang zum Internetportal: www.krankenhaus-report-online.de. D: 54,98 Euro / A: 56,60 Euro. ISBN: 978-3-7945-2972-8 (Print).

Das Krankenhaus als Risikofaktor? Diese provokative These ist Schwerpunkt der aktuellen Ausgabe des Krankenhaus-Report 2014, der jährlich in der Publikationsreihe des Wissenschaftlichen Instituts der AOK (WIdO) erscheint. Unterschiedliche Autoren befassen sich in Teil I mit dem Schwerpunktthema Patientensicherheit in der stationären Versorgung; zeigen Risiken auf, die eine Krankenhausbehandlung mit sich bringen kann und beschreiben, welches Ausmaß vermeidbare und nicht vermeidbare Schädigungen durch den Aufenthalt für den Patienten haben können. Rahmenbedingungen werden analysiert, Ursachen für unerwünschte Ereignisse diskutiert und Lösungsoptionen sowie Ansatzpunkte für eine Verbesserung der Patientensicherheit gesucht.

Krankenhausleistungen und Risiken einer Krankenhausbehandlung Im ersten Block geht es zunächst um Grundlegendes, wie die Definitionen der Patientensicherheit oder die Erläuterungen von Begrifflichkeiten. Krankenhausleistungen werden den verfügbaren Zahlen über das tatsächlich vorhandene Risiko einer Krankenhausbehandlung gegenübergestellt. So kommt Prof. Dr.

Max Geraedts von der

Univer-

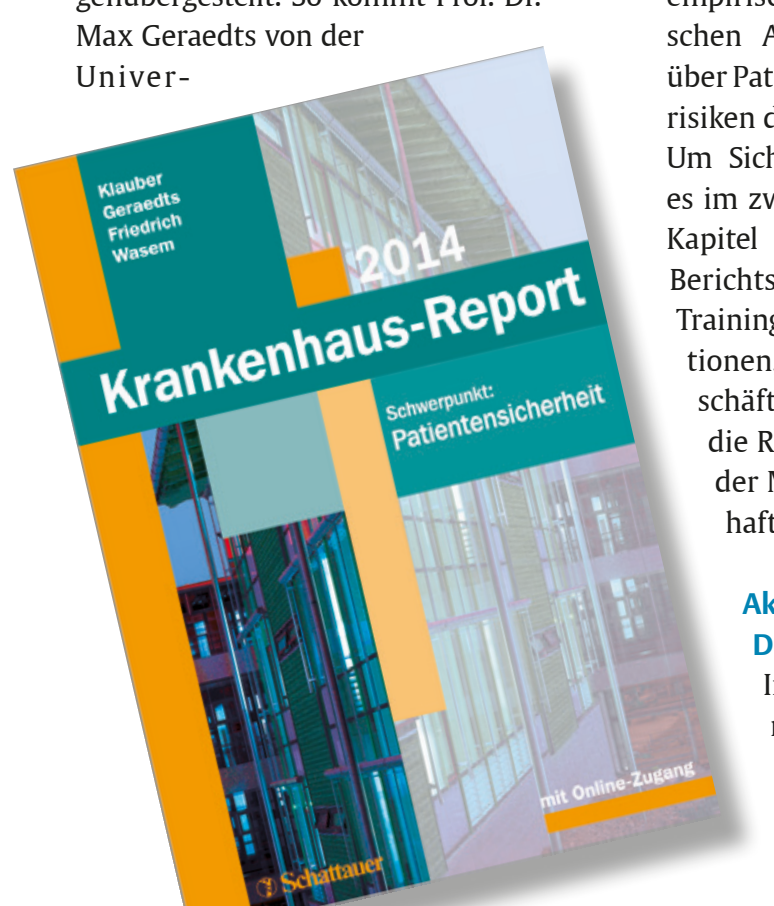

Aktuelle gesundheitspolitische Diskussion

Im Teil II des Reports, der wie immer der aktuellen gesundheitspolitischen Diskussion gewidmet ist, geht es um die „Krankenhausprivatisierungen in Deutschland und ihre Effekte“, die seit Beginn dieser Entwicklung kontrovers diskutiert werden.

Die Spezialisierung der Krankenhauslandschaft hat das Kapitel 16 „Zum Zusammenhang von Behandlungshäufigkeit und -ergebnis in der Hüftendoprothetik“ im Blick. Mehr als 150000 Patienten werden jährlich in Deutschland in über 1100 Krankenhäusern wegen einer Koxarthrose am Hüftgelenk operiert. In dem Beitrag wird der Zusammenhang von Behandlungshäufigkeit und Ergebnisqualität für diesen Eingriff anhand einer AOK-Analyse mit anonymisierten Abrechnungsdaten der Jahre 2008-2010 untersucht. Neben der Sterblichkeit und Revisions-Operationen wurden auch chirurgische Komplikationen, Thrombosen/Lungenembolien sowie Femurfrakturen mit berücksichtigt. Das Ergebnis ist wenig überraschend: höhere Fallzahlen bringen bessere Behandlungsqualität für alle untersuchten Komplikationen. Die gefundenen Unterschiede sind allerdings beträchtlich. Nach dieser Analyse hat das Fünftel der Krankenhäuser, die weniger als 45 Eingriffe im Jahr durchführen, nach Risikoadjustierung ein um 37\% erhöhtes Komplikationsrisiko.

\section{Statistik: trockener Stoff, aber solide Handlungsgrundlage}

Wie bei jeder Ausgabe endet auch diese Ausgabe des Krankenhaus-Report 2014 mit einem Statistikteil; ein umfassendes Kompendium von Analysen und Daten zur Entwicklung des Krankenhausmarktes. Insbesondere macht das Krankenhaus-Directory einen differenzierten Vergleich der einzelnen deutschen Kliniken anhand von Kennzahlen möglich.

Ergänzt wird dieses Angebot durch das Internetportal www.krankenhausreport-online.de. Trockener Stoff, aber eine solide Diskussions- und Handlungsgrundlage für Krankenhausmanager, Gesundheitspolitiker und -ökonomen.

Anne Marie Feldkamp, Bochum 